\section{EDUCATION}

Research, Inovoction and Solutions on-line
PSYCHOLOGY

I+D+i
Electronic Journal of Research

in Educational Psychology

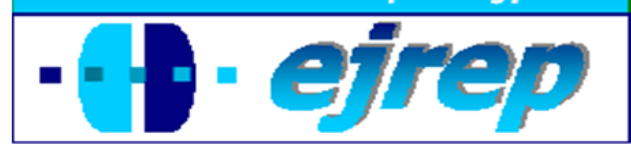

Editorial EOS

\title{
Estudio descriptivo de las estrategias de afrontamiento del bullying, en profesorado mexicano
}

\section{Alejandra Elizalde Castillo}

Universidad de las Américas, A.C. Ciudad de México

México

Correspondencia: Alejandra Elizalde Castillo. Av. Real del Country, 4. Lomas Country Club. Huixquilucán 52779, Estado de México. E-mail: aelizaldemx@yahoo.com

(C) Education \& Psychology $\mathrm{I}+\mathrm{D}+\mathrm{i}$ and Editorial EOS (Spain) 


\section{Resumen}

Introducción. Un problema frecuente y que se ha convertido en una situación estresante para los profesores es el bullying entre alumnos, actividad cada vez más prevalente y destructiva en las escuelas de México y el mundo. Los estilos de afrontamiento están relacionados con la manera como los profesores manejan las situaciones de bullying.

Método. El propósito de éste estudio consiste en describir las estrategias de afrontamiento, que utilizan los profesores de secundaria frente al bullying, dividiéndolos por cohortes. Participaron profesores de Secundaria $(\mathrm{N}=130)$ de 10 escuelas privadas de la Ciudad de México. Se utilizó el cuestionario de "Modos de afrontamiento al estrés de Lazarus y Folkman".

Resultados. De los resultados se extrae que las estrategias de afrontamiento utilizadas con mayor frecuencia frente al bullying son el afrontamiento dirigido al problema, apoyo social, confrontación y revaloración positiva. En relación al análisis por cohortes, se encontraron diferencias significativas por género, edad y por la información recibida sobre bullying.

Discusión y Conclusiones. A partir de los resultados se destaca la importancia de identificar las estrategias de afrontamiento utilizadas con mayor frecuencia en el manejo del bullying, tales como el dirigido al problema, el apoyo social, confrontación y revaloración positiva. Considerar dichas estrategias dentro de la cultura, principios, valores y creencias de cada escuela puede ser útil en el diseño de programas dirigidos a la prevención e intervención del bullying entre alumnos.

Palabras Clave: Bullying, estrés, estrategias de afrontamiento, profesores de secundaria.

Recibido: 14/12/09 Aceptación Inicial: 07/01/10 Aceptación Definitiva: 15/03/10 


\title{
Descriptive study about bullying' coping strategies among Mexican teachers
}

\begin{abstract}
Introduction. One frequent problem that teachers face, and that has become a stressful situation for them is bullying between students: an activity that has turned into a more prevalent and destructive situation in Mexican schools and in the rest of the world. Coping strategies are related to the way in which teachers handle bullying situations.

Method. The purpose of this study is to describe the way in which high-school teachers cope during bullying situations. One hundred and thirty high school teachers participated in 10 private schools in México City. Coping strategies were measured using a Mexican version of questionnaire "Ways of Coping Strategies". In order to obtain socio-demographical data, a questionnaire was specifically designed for this study.
\end{abstract}

Results. The data showed that the most frequent strategies used by teachers during bullying situations are planful problem solving, seeking social support, confrontive coping and positive reappraisal. In relation to cohorts important differences were found by gender, age and previous information on bullying.

Discussion y Conclusiones. This research highlights the importance of identifying highschool teachers frequent ways of copying during bullying situations, such as planful problem solving, social support confrontive coping and positive reappraisal. Considering such coping strategies within a culture, principles and beliefs of each school can be useful when designing programs for bullying between students.

Keywords: Bullying, stress, coping strategies, high-school teachers

Received: 12/14/09 Initial Acceptance: 01/07/10 Definitive Acceptance: 03/15/10 


\section{Introducción}

Durante las dos últimas décadas, el acrecentamiento de investigaciones que estudian el bullying ha dejado claro la universalidad de este fenómeno, si bien éste adquiere variaciones y matices particulares en cada cultura. En otras palabras, el bullying se da en todo el mundo y en todas las escuelas pero no de la misma forma. (Olweus, 2001; Smith, Schneider y Ananiadou, 2004).

Actualmente, en México cada vez son más frecuentes las noticias relacionadas con el tema de bullying, (Archundia, 2009a y 2009b; Fernández, 2009; Hernández, 2008; “Intentan prevenir”, 2009; Montaño, 2009; “Pública CDHDF”, 2008; Ortega, 2005). Los investigadores antes mencionados, sin duda coinciden en que este fenómeno es cada vez más recurrente, generalizado y se ha convertido en un asunto de ética social. Medina, en su estudio en México, (Primera Encuesta Media Superior de exclusión, intolerancia y violencia, 2008), señala que 7 de cada 10 niños han sido víctimas o testigos de bullying.

Por su parte, el uso del término anglosajón "bullying" (con el significado de torear), con profusión en el ámbito escolar, se encuentra con frecuencia en la literatura y en países de habla hispana como bullying, violencia escolar, intimidación, maltrato escolar, entre otros (Piñuel \& Oñate, 2006).

Diversas investigaciones (Aluede, Adeleke \& Akpaida, 2008; Benítez y Justicia, 2006; Beran, 2006; Brown, Birch y Kancheria, 2005; Craig y Pepler, 2007; Coloroso, 2004; Crothers \& Kolbert, 2008; De Zatarain, 2008; Farrington, 1993; Hunter y Boyle, 2004; Olweus, 1993, 2001; Scarpaci, 2006) han coincidido en que el bullying se produce cuando en repetidas ocasiones se ejerce violencia física, psicológica y/o social de una persona más poderosa hacia otra en estado de indefensión. Se ha establecido que una de las poblaciones más vulnerables es la de alumnos de secundaria que se encuentran en el inicio de la adolescencia y que en general las conductas de bullying suelen producirse entre los 10 y 14 años y van disminuyendo conforme los niños van haciéndose mayores. (Farrington, 1993; Harris y Petrie, 2003; Olweus, 1993).

Por otra parte, bullying es un proceso y dinámica grupal, como se puede observar en la figura 1, en la que se involucran muchos participantes y que generalmente ocurre en presencia 
de otros compañeros, quienes pueden adoptar varios roles, tales como: permanecer neutrales durante una situación de bullying, ayudar o apoyar al agresor o apoyar o consolar a la víctima. En la mayoría de los casos, el rol adoptado es el de apoyar la situación de bullying (Farrington, 1993; Harris y Petrie, 2003; Olweus, 1993).

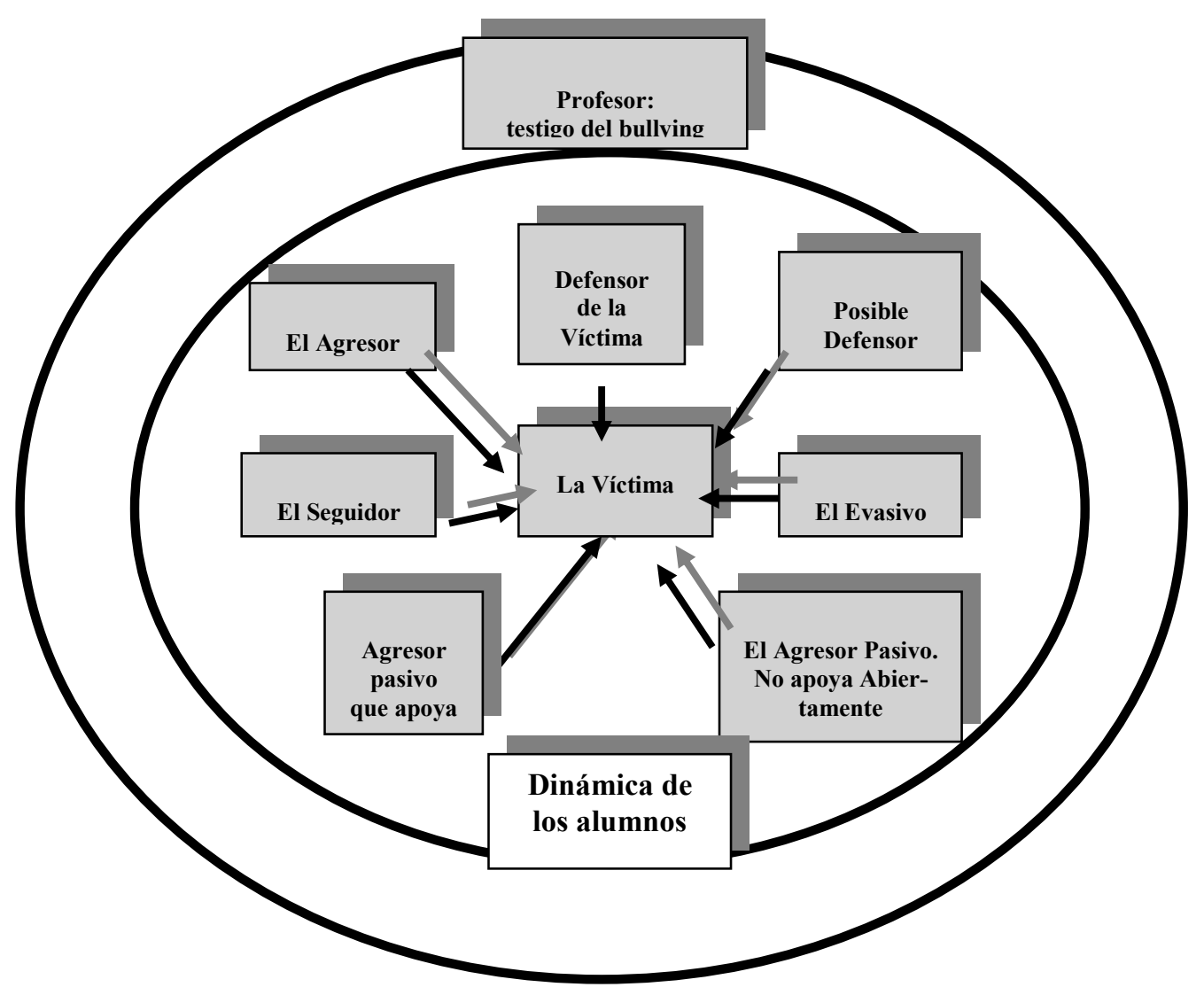

Figura 1. Dinámica del Bullying

Para Smith et al. (2004) y Trautmann (2008) los observadores juegan un papel muy importante en esta dinámica, ya que en algún momento de nuestras vidas, todas hemos sido testigos de algún acontecimiento del bullying. Existen dos universos de testigos, primero el de los alumnos y segundo los profesores y personal del colegio (ver figura 1). En este sentido, el profesor de secundaria tiene un papel fundamental como testigo o espectador del acoso, ya que se ha establecido que el observador es la audiencia del agresor. Los testigos o espectadores, como puede ser tanto el caso de los alumnos como de los profesores tienen un rol preventivo. Trautmann (2008) en su estudio señala que 30\% de los testigos han intentado ayudar a la víctima mientras que el 70\% no intentó intervenir porque "no era de su incumbencia". De acuerdo con los alumnos, los profesores no intervienen en situaciones de bullying, y sólo un 
tercio de los alumnos cree que a los profesores les interesa poner fin al bullying (Trautmann, 2008).

Las programas e intervenciones exitosas (Alcántara, 2009; Olweus, 2001; Smith.et al., 2004) están dirigidas en su mayoría a toda la comunidad educativa, (padres de familia, profesores, alumnos, personal administrativo entre otros) y tienen la función de influir e intervenir con distintas actividades en todo el ambiente escolar e involucrar a individuos, víctimas, agresores, grupos de pares, salas de clases, profesores, administración, familias en general y familias de víctimas y agresores.

El mayor éxito de este tipo de enfoque sugiere que hay factores externos al individuo y que tienen que ver con procesos de interacciones sociales, sin embargo, hay barreras que limitan su efectividad. La implementación varía en forma significativa y el éxito dependería de la duración del programa, del compromiso y entrenamiento de los profesores y autoridades del colegio (Olweus, 2001; Smith et al.,2004; Trautmann, 2008). Este abordaje refleja la idea de que el bullying es una interacción entre un agresor y una víctima pero ambas pertenecen a un contexto social ecológico, esta postura evita el estigma de agresor-víctima (Bronfenbrenner, 1979; Moen, Elder y Lusher, 1995; Ramírez y Justicia; 2006).

Hoy en día, los profesores además de promover el desempeño académico, tienen responsabilidades que incluyen el manejo de conductas y socialización de sus estudiantes. En la mayoría de las ocasiones, los profesores que trabajan de cerca con sus alumnos están en mejores condiciones para identificar problemas de bullying entre sus alumnos (Bardick \& Bernes, 2008), sin embargo y a pesar de la gravedad del problema, son muchos los profesores que no se involucran en la solución del fenómeno (Benítez, García y Fernández, 2007).

De acuerdo con Beran (2006) y Yoon (2004) una conducta que los profesores encuentran cada vez con más regularidad en sus clases es el bullying entre alumnos, lo que significa menos tiempo dedicado a lo académico (Beran, 2006; Yoon 2004). Diversos estudios (Avilés \& Monjas, 2005; Ellis y Shute, 2007; Yoon et al., 2003) han encontrado existe una falta de intervención por parte de los profesores en situaciones de bullying.

Algunos interrogantes que se plantean en esta investigación, son las siguientes: ¿Cómo afrontan los profesores de secundaria el bullying entre sus alumnos? ¿Existen diferencias de 
afrontamiento entre los profesores hombres y mujeres? ¿Existen diferencias de acuerdo a su edad? Autores como Elder y Giele (1998); Moen, Elder y Lüshcer (1995); Settersten (1999) coinciden en que la edad y el tiempo histórico por sí solas son variables vacías, pero si se entienden como parte del modelo del curso de la vida ${ }^{1}$ estaremos en mejores condiciones de entender cómo el ambiente, cultura y cambios sociales han afectado de alguna manera a una cohorte. El sexo y la edad de los profesores, resultan ser variables importantes desde la perspectiva del individuo y estructuras sociales, debido a que funcionan como dimensiones con las cuales se pueden mapear las expectativas sociales y culturales sobre la experiencia y los roles $\mathrm{y}$, en este sentido saber con mejor precisión cómo pueden los profesores manejar las situaciones de bullying con mayores probabilidades de éxito.

Una vez planteado el problema y el camino por aclarar y formular los conceptos centrales, se puede afirmar que el propósito de este estudio es describir las estrategias de afrontamiento que los profesores de secundaria utilizan frente al bullying con la finalidad de estar en mejores condiciones para intervenir eficientemente frente a estas situaciones.

En este estudio se consideran las estrategias de afrontamiento desde el enfoque de las emociones en sus dimensiones cognitiva, emocional y motivacional (Campos, Campos, y Barros, 1989; Frijda, 1986; Gross y Thompson, 2007; Lazarus, DeLongis, Folkman y Gruen, 1985; Lazarus, 1991a; Lazarus, 1991b; Lazarus, 1999; Orange, 1995). De acuerdo con Lazarus (1984), las estrategias de afrontamiento son los esfuerzos cognoscitivos y conductuales cambiantes para manejar las demandas específicas externas y/o internas, evaluadas como excedentes o desbordantes de los recursos del individuo. En este sentido, las emociones como estados relacionales se originan cuando el individuo está en una situación específica que valora como relevante para lograr sus metas, y le atribuye un significado que puede o no cambiar con el tiempo, modificando nuevamente sus emociones. (Campos, Campos y Barret, 1989).

En el estudio de Piñuel (2006), se reporta que el 73\% de los profesores se encuentran estresados por la violencia procedente de los salones de clase. Lazarus, (1999) estableció que

\footnotetext{
1 "El curso de la vida" se refiere a los roles de transición relacionados con la edad que son "creados socialmente, reconocidos socialmente y compartidos". Se centra en las experiencias de grupo y en las fuerzas sociales que estructuran la vida. En este sentido las vidas humanas no son cíclicas, los individuos no regresan al mismo punto durante el desarrollo de la vida, ni terminan en las mismas circunstancias en que empezaron. (Elder y Giele, 1998; Moen, Elder y Lüscher, 1995; Settersten, 1999).
} 
un evento estresante traumático es un proceso dinámico en dónde las personas experimentan emociones, estados de ánimo o pensamientos contradictorios y tienen estrategias de afrontamiento muy complejas. El afrontamiento es un proceso, cambiante con demandas y conflictos específicos (Lazarus, 1999). Los tres aspectos principales del proceso para afrontar en los profesores son:

a) El que hace referencia a las observaciones y valoraciones relacionadas con lo que el profesor realmente piensa o hace en contraposición con lo que generalmente hace o haría en determinadas condiciones.

b) Lo que el profesor realmente piensa o hace, es analizado dentro de un contexto específico.

c) El proceso de afrontamiento implica un cambio en los pensamientos y actos a medida que la interacción va desarrollándose.

El afrontamiento comienza desde la evaluación inicial que un profesor haga de una situación de bullying (intensidad, duración y frecuencia, así cómo las características de los involucrados, como la edad, género, entre otros) y el significado que le de a la situación para posteriormente evaluar con qué recursos cuenta y necesita de acuerdo a la demandas, para entonces seleccionar una estrategia de afrontamiento y llevarla a cabo. Lazarus (1999) plantea las siguientes funciones del afrontamiento que son los objetivos que persigue cada estrategia de afrontamiento, mientras que los resultados de éste se refieren al efecto que tiene cada estrategia:

- Afrontamiento dirigido al problema: Sus funciones consisten en tratar de disminuir el estrés haciendo cambios conductuales, en cambiar o modificar la fuente del estrés, cambiar la relación persona-ambiente, dar soluciones cognoscitivas y conductuales y estrategias emocionales como la confrontación o planteamiento de solución al problema

- Afrontamiento dirigido a la emoción: sus funciones son reducir el estrés emocional manteniendo un estado emocional satisfactorio, cambiar el estado emocional que acompaña la situación estresante, cambiar la relación emoción-situación estresante y estrategias de afrontamiento como escape, evitación y distanciamiento (Lazarus, 1999; Sotelo, 1996). 
En el estudio de Góngora y Reyes (1999) sobre las principales formas de enfrentamiento a diferentes situaciones de problemas, en una población mexicana de yucatecos, encontraron que el afrontamiento más utilizado es el directo-revalorativo, que se refiere a hacer algo para resolver el problema y tratar de aprender lo positivo. El segundo modo de afrontamiento más utilizado es el emocional-negativo, que se refiere a tener un sentimiento o emoción que no lleva directamente a la solución del problema (Góngora y Reyes, 1999).

Por su parte el apoyo social como estrategia de afrontamiento por parte de los profesores, compañeros, apoyo de la escuela y de los padres ayuda a moderar la relación entre la victimización y el estrés causado por el bullying (Davidson y Kilpatrick, 2007).

\section{Método}

\section{Participantes}

La metodología de investigación fundamentalmente consistió en un diseño no experimental, transversal, de tipo descriptivo. Esto se fundamenta en que el presente estudio parte de la recolección de datos en un momento dado, y de la descripción de las variables.

Participaron 130 profesores de secundaria seleccionados de forma no probabilísticas durante los meses de Mayo-Junio del 2009 en escuelas privadas de la zona metropolitana de México.

\section{Instrumentos}

Se utilizó el cuestionario de modos de afrontamiento al estrés elaborado por Folkman y Lazarus (Ways of copying, 1988) y adaptado por Sotelo (1999) para la población mexicana, con un valor $\alpha=.85$.

El instrumento tiene un formato de escala tipo Likert con cuatro opciones de respuesta: nada, en alguna medida, casi siempre y siempre. El cuestionario incluyó un total de 66 reactivos. Se adaptaron las instrucciones del cuestionario para los fines de esta investigación. 
El cuestionario se conforma de 8 dimensiones:

1. Afrontamiento dirigido al problema: acciones conductuales que van dirigidas a confrontar activamente el problema y tienen efectos positivos en el bienestar de la persona, $(\alpha=.68)$. Por ejemplo, "Desarrollé un plan y lo seguí".

2. Confrontación: describe los esfuerzos para modificar la situación y sugiere un cierto grado de riesgo, agresión y hostilidad. $(\alpha=.70)$. Por ejemplo: “Traté de buscar al responsable del acoso".

3. Distanciamiento: El profesor evade todo contacto con el problema de bullying y su solución, para de esta manera reducir la angustia que éste le provoca $(\alpha=.61)$. Por ejemplo, "Seguí adelante como si no hubiera pasado nada".

4. Autocontrol: Describe los esfuerzos del profesor para regular sus sentimientos y acciones. Guardan para sí mismos los afectos y sentimientos causados por el problema $(\alpha=.70)$. Por ejemplo, "Intenté no actuar impulsivamente o hacer lo primero que se me ocurriera".

5. Búsqueda de apoyo social: Describe esfuerzos para buscar apoyo informativo; búsqueda de una red de apoyo social que provea de solidaridad, consejo, atención, información, auxilio y protección $(\alpha=.76)$. Por ejemplo, "Busqué la ayuda de un profesional".

6. Aceptar responsabilidad: Darse cuenta del rol que tiene uno en el problema para entonces resolverlo $(\alpha=.66)$ Por ejemplo, "me prometí que las cosas serían diferente la próxima vez".

7. Revaloración positiva: Describe los esfuerzos para crear resultados positivos enfocándose al crecimiento personal; manifestando una sensación positiva de su propio valor, una vista positiva hacia los demás y en general una sensación de optimismo $(\alpha=.79)$. Por ejemplo, "Me sentí más fuerte después de resolver el problema, que antes de tenerlo".

8. Evitación: Es el grado en que los sujetos usan conductas para promover o poner en riesgo la salud e incrementar o disminuir su bienestar físico; o bien, pueden optar por la creación de fantasías para mitigar la realidad poco placentera $(\alpha=.72)$. Por ejemplo, "Me negué a creer lo que estaba pasando".

\section{Procedimiento}

Primero se procedió a verificar con 12 jueces expertos (Psicólogos con nivel de estudios de maestría) las instrucciones que se adaptaron para la aplicación del cuestionario y se 
hicieron las modificaciones necesarias. Segundo, mediante un muestreo por conveniencia se contactaron a los directivos, coordinadores o departamentos de psicología de las escuelas en la zona metropolitana de la Ciudad de México. Se les hizo la invitación y se les entregó una carta de la Universidad las Américas, la cual incluyó la justificación, objetivos y procedimiento del estudio. La aplicación del instrumento fue administrado bajo condiciones de confidencialidad, asegurando a los profesores el anonimato.

\section{Análisis Estadístico}

Hecho lo anterior se llevó a cabo el análisis de datos utilizando el SPSS versión 17.0. Se realizó un análisis descriptivo para las variables sociodemográficas, y después se procedió a hacer el análisis del estudio. Inicialmente se obtuvieron los puntajes relativos, para cada dimensión, que se refieren a la proporción con la que los participantes utilizaron estas estrategias, después se compararon las medias de dichos puntajes para saber cuáles son las que utilizan con mayor frecuencia.

Se procedió a realizar un análisis no paramétrico para obtener diferencias por sexo y de los profesores que han recibido información de bullying y los que no la han recibido. Posteriormente se realizó la prueba de ANOVA de una vía para comparar los grupos por edad.

\section{Resultados}

Se realizó un análisis descriptivo de las variables sociodemográficas que fueron utilizadas para el análisis: El 100\% (N=130) esta conformado por profesores mexicanos que imparten clases en secundaria en escuelas privadas de la Ciudad de México. En lo que se refiere al sexo el $66.4 \%(n=87)$ fue conformado por mujeres y el $33.6 \%(n=43)$ por hombres. Los profesores que han recibido información de bullying son el $77.7 \%(n=99)$ mientras que el $28.2 \%(\mathrm{n}=39)$ no ha recibido la información.

Como se puede observar en la tabla 1 las estrategias más utilizadas por los profesores son: el afrontamiento dirigido al problema, apoyo social, revaloración positiva y autocontrol, siendo las menos frecuentes la evitación, distanciamiento y aceptar responsabilidad. 
Tabla 1. Estrategias utilizadas con mayor frecuencia

\begin{tabular}{lcccc}
\hline Estrategias de Afrontamiento $^{*}$ & $\mathbf{X}$ & $\mathbf{S x}$ & $\mathrm{X}^{2}$ & $\mathrm{p}$ \\
\hline Afrontamiento dirigido al problema & 1.53 & .56 & 63.1 & .000 \\
Apoyo Social & 1.44 & .61 & 50.0 & .000 \\
Revaloración positiva & 1.37 & .77 & 26.0 & .166 \\
Autocontrol & 1.28 & .56 & 56.0 & .000 \\
Confrontación & 1.27 & .48 & 71.0 & .000 \\
Aceptar responsabilidad & .82 & .60 & 50.0 & .000 \\
Distanciamiento & .75 & .48 & 55.2 & .000 \\
Evitación & .36 & .29 & 46 & .000 \\
\hline *valor mínimo $=0$ & valor máximo $=3$ & & & \\
\hline
\end{tabular}

En lo que respecta a las estrategias de afrontamiento entre hombres y mujeres se pueden observar en la tabla 2 diferencias significativas en la dimensión de apoyo social, $(p=.004)$ y revaloración positiva ( $p=.002)$ siendo las mujeres las que utilizan más esta estrategia. No se encontraron diferencias significativas en las demás dimensiones.

Tabla 2. Diferencias en torno a las estrategias de afrontamiento por sexo

\begin{tabular}{lcccccc}
\hline \multicolumn{1}{c}{ Estrategias de Afrontamiento } & Mujeres & \multicolumn{2}{c}{ Hombres } & & & \\
& $\mathbf{X}$ & $\mathbf{X}$ & $\mathbf{X}^{2}$ & $\mathbf{g l}$ & $\mathbf{p}$ \\
\hline Afrontamiento dirigido al problema & 1.57 & 1.46 & 21.7 & 16 & .151 \\
Apoyo Social & 1.54 & 1.23 & 28.9 & 16 & .024 \\
Revaloración positiva & 1.44 & 1.24 & 43.0 & 20 & .002 \\
Autocontrol & 1.30 & 1.24 & 15.3 & 17 & .572 \\
Confrontación & 1.24 & 1.34 & 16.4 & 14 & .287 \\
Aceptar Responsabilidad & .79 & .89 & 6.12 & 10 & .805 \\
Distanciamiento & .72 & .82 & 16.6 & 12 & .162 \\
Evitación & .37 & .35 & 13.8 & 9 & .128 \\
\hline
\end{tabular}

En relación a la edad de los profesores se encontraron diferencias significativas en las siguientes dimensiones: (ver tabla 3) aceptar responsabilidad ( $p=.025)$ y confrontación $(p=.039)$. 
Tabla 3. Diferencia en torno a las estrategias de afrontamiento por edad

\begin{tabular}{|c|c|c|c|c|c|c|c|}
\hline \multirow[t]{2}{*}{ Estrategias de Afrontamiento } & \multicolumn{3}{|c|}{$\begin{array}{c}\text { EDAD } \\
X\end{array}$} & \multirow[b]{2}{*}{51 ó más } & \multirow[b]{2}{*}{$\mathbf{F}$} & \multirow[b]{2}{*}{ gl } & \multirow[b]{2}{*}{ p } \\
\hline & 20-30 & $31-40$ & 41-50 & & & & \\
\hline Afrontamiento dirigido al problema & 1.40 & 1.44 & 1.54 & 1.80 & 2.42 & 120 & .069 \\
\hline Apoyo Social & 1.27 & 1.47 & 1.42 & 1.61 & .95 & 125 & .418 \\
\hline Revaloración positiva & 1.13 & 1.35 & 1.32 & 1.73 & 1.85 & 116 & .141 \\
\hline Autocontrol & 1.13 & 1.24 & 1.25 & 1.51 & 1.62 & 119 & .186 \\
\hline Confrontación & 1.11 & 1.20 & 1.32 & 1.53 & 2.87 & 121 & .039 \\
\hline Aceptar Responsabilidad & 1.03 & .71 & .72 & 1.10 & 3.22 & 121 & .025 \\
\hline Distanciamiento & .82 & .72 & .69 & .86 & .739 & 122 & .531 \\
\hline Evitación & .38 & .34 & .36 & .41 & .29 & 116 & .827 \\
\hline
\end{tabular}

Con respecto a la información de bullying, se pueden observar en la tabla 4 diferencias significativas en las siguientes dimensiones: apoyo social $(p=.011)$, afrontamiento dirigido al problema $(p=.034)$ y aceptar responsabilidad $(p=.011)$.

Tabla 4. Diferencia en torno a las estrategias de afrontamiento en profesores que han recibido información de bullying y los que no

\begin{tabular}{lcccccc}
\hline \multicolumn{1}{|c}{ Estrategias de Afrontamiento } & $\begin{array}{c}\text { Si han } \\
\text { recibido } \\
\text { información } \\
\mathbf{X}\end{array}$ & $\begin{array}{c}\text { No han } \\
\text { recibido } \\
\text { información }\end{array}$ & $\mathbf{X}^{2}$ & $\mathrm{gl}$ & $\mathbf{p}$ \\
\hline Afrontamiento dirigido al problema & 1.61 & 1.34 & 27.6 & 16 & .034 \\
Apoyo Social & 1.57 & 1.13 & 31.8 & 16 & .011 \\
Revaloración positiva & 1.47 & 1.14 & 14.3 & 20 & .813 \\
Autocontrol & 1.34 & 1.14 & 25.3 & 17 & .087 \\
Confrontación & 1.34 & 1.10 & 25.3 & 14 & .067 \\
Aceptar Responsabilidad & .87 & .69 & 22.9 & 10 & .011 \\
Distanciamiento & .73 & .80 & 17.5 & 12 & .131 \\
Evitación & .39 & .30 & 19.85 & 9 & .106 \\
\hline
\end{tabular}




\section{Discusión y Conclusiones}

Se ha establecido que los profesores influyen día a día en la vida de los estudiantes, primero reconociendo los incidentes del bullying y segundo implementando estrategias de intervención o lineamientos para su manejo (Benítez et al., 2007; Mishna, Scarcello, Pepler y Wiener, 2005). Las investigaciones (Áviles y Monjas, 2005; Ellis y Shute, 2007; Yoon et al., 2003) han encontrado, que existe una falta de intervención por parte de los profesores en situaciones del bullying. EL objetivo de este estudio fue conocer las estrategias de afrontamiento frente al bullying, en profesores de secundaria en escuelas particulares de la Ciudad de México.

Los hallazgos de este estudio confirman que existe una falta de utilización de estrategias en el manejo del bullying, por parte de los profesores, ya que la frecuencia con la que las utilizan de manera global es del 36\%. Una posible explicación puede ser que los profesores no perciben el bullying o no lo consideran serio. Para futuras investigaciones sería pertinente corroborar esta hipótesis. La falta de intervención en el manejo del bullying es un indicador clave, para resolver estas situaciones, ya que habría que fomentar cursos de capacitación para que los profesores sean más proactivos y se involucren más en este tipo de problemas, ya que definitivamente forman parte de ellos.

Tal como se refleja en el estudio de Góngora y Reyes (1999), en donde predominan el afrontamiento para resolver el problema y tratar de aprender lo positivo, en este estudio, se encontró que las estrategias de afrontamiento más utilizadas frente al bullying son: el afrontamiento dirigido a la resolución del problema, apoyo social y revaloración positiva

El apoyo social, que se refiere a los esfuerzos para buscar apoyo informativo, búsqueda de una red de apoyo social que provea de solidaridad, consejo, atención, información, auxilio y protección(Lazarus, et al.,1985; Lazarus,1991a; Lazarus, 1991b) resultó ser la estrategia más utilizada por las mujeres y las más utilizada por profesores que han recibido información de bullying.

De acuerdo con la literatura (Davidson y Kilptatrick, 2007; Hirschstein, Van Schoiack, Frey, Snell y MacKenzie, 2007; Olweus, 1993) el apoyo social es un regulador importante para los profesores en situaciones de bullying, misma que puede ser implementada en pro- 
gramas preventivos y para víctimas de estas situaciones, ya que ayuda a amortiguar la relación entre la victimización y el estrés causado por el bullying. Se puede potenciar dicho apoyo social, apoyando a los alumnos para desarrollar conductas pro-sociales, y enseñando habilidades socio-emocionales tanto a profesores como a alumnos. Para futuras investigaciones sería pertinente examinar la percepción que tienen las víctimas y la importancia del tipo de apoyo social que perciben en sus vidas.

La estrategia de revaloración positiva es la tercera estrategia más utilizada en esta muestra de profesores sobre todo por mujeres. Estos hallazgos coinciden con lo reportado por Góngora y Reyes (1999) en una población Yucateca, sin embargo habrá que potenciar esta estrategia para que lleve a la resolución del problema, ya que podría ser que resulte una conducta no adaptativa y en este sentido se encuentre lejos de resolver el problema. Para futuras investigaciones sería útil examinar con mayor precisión los tipos de revaloraciones positivas que ayudan a la resolución de dichas situaciones.

En relación con la edad de los profesores, se encontró que mientras mayores sean los profesores utilizan más la confrontación como estrategia. Por su parte los profesores que tienen entre 20 y 30 años de edad y mayores de 50 años aceptan más la responsabilidad del bullying que los que se encuentran entre 31 y 51 años de edad. Será interesante continuar con esta línea de trabajo que considere otros factores como personalidad, familia, para entender mejor estas diferencias.

Una limitación que encontramos es que a pesar de que el instrumento ya fue validado en población mexicana, es necesario hacer una continua validez ecológica del mismo, ya que el contexto escolar tiene variaciones con otras poblaciones (Bronfenbrenner, 1979; Moen, Elder y Lusher, 1995).

Para futuras investigaciones sería interesante saber qué opinión tienen los alumnos, sobre las estrategias que los profesores dicen tener con los alumnos y hacer análisis de las estrategias utilizadas por profesores que hayan sido víctimas, testigos o agresores de bullying. 
Puesto que los profesores finalmente son uno de los agentes de cambio en la prevención del bullying este estudio nos ha abierto un camino fértil para que los maestros tengan a su alcance nuevos caminos de aprendizaje. 


\section{Referencias}

Alcántara, M. (2009). Prevenir el bullying desde la familia. México.: Editorial Minos.

Aluede,O., Adeleke,F., y Akpaida, A.J. (2008). A review of the extent, nature, characteristics and effects of bullying behaviour in schools. Journal of Instructional Psychology, 35, 151-158.

American Psychological Association, Council of Editors. (1999). Manual de estilo de Publicaciones de la American Psychological Association. México D. F.: Editorial El Manual Moderno.

Archundia, M. (2009a, 22 de enero). Bullying: cuando la violencia es juego. Diario El Universal, p.C2.

Archundia, M. (2009b, 11 de junio). "Bullying” inicio de la delicuencia: especialista. Diario El Universal, p. A13.

Avilés, J.M y Monjas, I. (2005) Estudio de Incidencia de la Intimidación y el Maltrato Entre Iguales en la Educación Secundaria Obligatoria mediante Cuestionario CIMEI. Anales de Psicología, 21, 27-41.

Bardick, D.A \& Bernes. B.K. (2008) A framework for assessing violent behavior in Elementary schools. Children \& Schools, $30,83-91$.

Benítez, J.L., García. B.A \& Fernández, M.F. (2007). Conocimiento, percepciones y actitudes hacia el maltrato entre iguales entre el profesorado en activo y los docentes en formación. Psicología Educativa, 13, 151-166.

Benítez, J. L. \& Justicia F. (2006). El maltrato entre iguales: descripción y análisis de un fenómeno. Electronic Journal of Research in Educational Psychology, 4(2), 151-170.

Beran, N.T. (2006). Preparing teachers to manage school bullying: The hidden curriculum. The Journal of Educational Thought, 40, 119-128.

Boszormengy-Nagy, I \& Spark, G. (2008). Lealtades Invisibles. Reciprocidad en terapia familiar intergeneracional. Buenos Aires.: Amorrortu editors.

Bronfenbrenner, U. (1979). The Ecology of Human Development. New York.: The Harvard University Press.

Brown, L.S., Birch, A.D y Kancheria, V. (2005). Bullying Perspectives: Experiences, Attitudes, and recommendations of a 9- to 13- year olds attending health education Centers in the United States. The Journal of School Health, 75, 384-92. 
Campos, J.J., Campos, G.R., y Barret, C.K. (1989). Emergent Themes in the Study of Emotional Development and Emotion Regulation. Developmental Psychology, 25, 394402.

Coloroso, B. (2004). The Bully, the Bullied, and the Bystander. New York.: Harper Resource.

Craig, M.W y Pepler J.D. (2007). Understanding bullying: from research to practice. Canadian Psychology, 48, 86-88.

Crothers, M.L., y Kolbert, B.J. (2008). Tackling a problematic behavior management issue: Teacher's intervention in childhood bullying problems. Intervention in School and Clinic, 43, 132-139.

Davidson. M.L y Kilpatrick. M. (2007). Social Support as a Moderator Between Victimization and Internalizing-Externalizing Distress From Bullying. School Psychology Review, 36, 383-405.

De Zataráin, F. (2008) Acoso escolar. México.: Editorial Jus.

Elder, G. y Giele, J. (1998). Methods of Life Course Research. U.S.A.: Sage Publications.

Ellis, A.A., \& Shute, R. (2007). Teacher responses to bullying in relation to moral Orientation and seriousness of bullying. British Psychological Society, 77, 649-663.

Farrington, D. (1993). Understanding and preventing bullying. Crime and Justice, 17, 381458.

Fernández, M, J. (2009, 3 de julio). Lo que no dijeron en las campañas. Diario El Excelsior.

Folkman, S y Lazarus. S.R. (1988). Ways of Copying Questionnaire Sampler Set. Consulting Psychologist press.

Framo, L.J. (1996). Familia de origen y psicoterapia. Un enfoque intergeneracional. Barcelona.: Paidós Terapia Familiar.

Frijda, N. (1986). The Emotions. New York.: Cambridge University Press.

Góngora, E y Reyes, I. (1999). La Estructura de los Estilos de Enfrentamiento: Rasgo y Estado en un Ecosistema Tradicional Mexicano. Revista Sonoriense de Psicología, 13, 2 14.

Gross, J. y Thompson, R. (2007). Emotion Regulation. En J.J Gross (Eds.), Handbook Of Emotion Regulation (pp. 3-24). New York.: The Guilford Press.

Hernández, M. (2008, 16 de noviembre). Enseñan a combatir el "bullying”. Reforma, Ciudad, pp.1.

Hirschstein, K.M , Van Schoiack, L, Frey, L, Snell L.J y MacKenzie, P.E. (2007). Walking the Talk in Bullying Prevention: Teacher Implementation Variables Related to intial Impact the Steps to Respect Program. School Psychology Review, 36, 3-21. 
Hunter, C.S y Boyle ,M.J. (2004). Appraisal and coping strategy use in victims of school bullying. British Journal of Educational Psychology, 74, 83-107.

Intentan prevenir violencia estudiantil (2009, 23 de abril). Reforma/Staff.

Lazarus, S.R., DeLongis A., Folkman.S., y Gruen, R. (1985). Stress and Adaptational Outcomes. American Psychologist, 40, 770-779.

Lazarus, S.R. (1991a). Progress on a Cognitive-Motivational-Relational Theory of Emotion. American Psychologist, 46, 819-834.

Lazarus, S.R. (1991b). Cognition and Motivation in Emotion. American Psychologist, 46, 354-367.

Lazarus, S.R. (1999). Stress and Emotion. New York.: Spring Publishing.

Medina, P (2008, 20 de octubre). Terror en las aulas. Diario El Excelsior, Página Nacional.

Mishna, F., Scarcello, I., Pepler, D \& Wiener, J. (2005). Teachers' understanding of Bullying. Canadian Journal of Education, 28, 718-738.

Moen, P, Elder, G., y Lüscher, K. (1995). Examining Lives In Context. Washington, D.C.: American Psychological Association,

Montaño, T (Sábado 15 de agosto de 2009). "BULLYING”, guerra, entre mujeres. El Universal, pp. A11.

Olweus, D. (1993). Bullying at School. Australia.: Blackwell Publishing.

Olweus, D. (2001). Bullying at school: Tackling the problem. Organization Economic Cooperation and Development, 25, 24-26.

Orange, D. (1995). Emotional Understanding. Studies in Psychoanalytic Epistemology. New York.: Guilford Press.

Ortega, B. S, Ramírez, M. A., y Castelán, C. A. (2005). Estrategias para prevenir y atender el maltrato, la violencia y las adicciones en las escuelas públicas de la Ciudad de México.

Piñuel, I. (2006). Estudio Cisneros VIII Violencia contra Profesores en la enseñanza pública de la comunidad de Madrid . Extraído el 10 de marzo de 2009 de: htpp//www.acosoescolar.com/Inicio/INFORME \%CISNEROS\%20VIII.pdf.

Piñuel, I y Oñate, A. (2006). Acoso y Violencia Escolar. Madrid.: TEA Ediciones.

Pública CDHDF cartilla sobre violencia escolar. (2008, 19 de septiembre) Extraído el 14 de abril de 2009 de: htpp//www.notimex.com.mx.

Ramírez, S y Justicia F. (2006). El maltrato entre escolares y otras conductas problemas para la convivencia. Electronic Journal of Research in Educational Psychology, 4(2), 265290. 
Sánchez, C. (2009, 5 de octubre). Usan la violencia para socializar en la "secu”. Diario El Universal, Sociedad, p.15.

Settersten. A. R. (1999). Lives in Time and Place. New York.: Baywood Publishing Company.

Scarpaci, T.R. (2006). Bullying: Effective strategies for its prevention. Kappa Delta Pi Record, 42, 170-174.

Smith, J.D ., Schneider, H.B.,Smith, K.P y Ananiadou, K. ( 2004). The effectivness of wholeschool antibullying programs: A synthesis of evaluation research. School Psychology Review, 33, 547-560.

Sotelo, C.M. (1999). Traducción y estandarización del cuestionario modos de afrontamiento al estrés de Lazarus y Folkman, para una población de adolescentes mexicanos. Tesis de Licenciatura, UNAM, Facultad de Psicología.

Trautmann, A. (2008). Maltrato entre pares o “bullying." Una visión actual. Revista Chilena de Pediatría, 79, 13-20.

Yoon, S.J. (2004). Predicting teacher intervention in bullying situations. Education \& Treatment of Children. 27, 37-45. 